\title{
MIMO Passive Control Systems Are Not Necessarily Robust
}

\author{
Jesús U. Liceaga-Castro, ${ }^{1}$ Irma I. Siller-Alcalá, ${ }^{1}$ Eduardo Liceaga-Castro, ${ }^{2}$ \\ and Luis A. Amézquita-Brooks ${ }^{2}$ \\ ${ }^{1}$ Departamento de Electrónica, Universidad Autónoma Metropolitana-Azcapotzalco, 02200 Ciudad de México, DF, Mexico \\ ${ }^{2}$ CIIIA-FIME, Universidad Autónoma de Nuevo León, 66451 Monterrey, NL, Mexico
}

Correspondence should be addressed to Jesús U. Liceaga-Castro; julc@correo.azc.uam.mx

Received 8 May 2015; Revised 22 June 2015; Accepted 10 September 2015

Academic Editor: Shengwei Mei

Copyright (C) 2015 Jesús U. Liceaga-Castro et al. This is an open access article distributed under the Creative Commons Attribution License, which permits unrestricted use, distribution, and reproduction in any medium, provided the original work is properly cited.

Via several cases of study it is shown that a passive multivariable linear control system, contrary to its single input single output counterpart, may not be robust. Moreover, it is shown that lack of robustness can be exposed via the multivariable structure function.

\section{Introduction}

The design of controllers for multivariable systems (MIMO) satisfying robustness conditions may be a long and tiresome procedure [1]. Many well-known methodologies had been proposed in order to solve this problem such as LQG, $H_{\infty}$, and QFT. More recently, dissipativity which is related to the conservation, dissipation, and transport of energy is an open loop property that has been used to develop a framework for the design and analysis of control systems. In particular, passive analysis focuses on systems which dissipate energy. This open loop characteristic presents great advantages in the design of robust control systems.

For the case of a linear time invariant SISO system described by a rational transfer function $G(s)=N(s) / D(s)$ is passive if it satisfies the following [2]:

(i) $G(s)$ is stable.

(ii) $\mathbb{R}[G(j \omega)] \geq 0 \forall \omega \in(-\infty, \infty)$.

These conditions can be translated in terms of the classical control theory as follows: a rational SISO transfer function $G(s)=N(s) / D(s)$ is passive if

(i) $G(s)$ is stable and minimum phase,

(ii) $G(s)$ has a relative degree $\leq 1$,

(iii) $G(s)$ behaves like a lead phase filter,

(iv) the Nyquist plot of $G(j \omega)$ lies in the right half plane of the complex plane $\forall \omega \in(-\infty, \infty)$.
That is, passive SISO systems are robust because they have gain and phase margins of $\mathrm{Mg} \rightarrow \infty \mathrm{dB}$ 's and $\mathrm{Mp} \geq 90^{\circ}$, respectively. Moreover, thanks to this characteristic the parallel, series, or feedback interconnection of two passive systems is also robust. In this context, in general, the strategy for passive control systems is divided in two steps: the passivation of the process and the design of a passive controller-normally a PID controller-based on the amended or passivized process. Also, in order to transform a nonpassive SISO system into a passive one, three methods are normally applied: pre- or postcompensation, feedback, and feed-forward $[3,4]$.

One may cast the conjecture on the existence of these frequency domain properties for linear MIMO systems. If this conjecture proves to be true, the robust design and assessment of multivariable control could be obtained in a similar manner as in SISO systems.

A square linear time invariant MIMO system can be represented by $y(s)=G(s) u(s)$, where $G(s) \in \mathbb{C}^{m x m}$ is a rational transfer function matrix; $u(t) \in \mathbb{R}^{m}$ is the input; and $y(t) \in \mathbb{R}^{m}$ the output. Assuming all the poles of $G(s)$ are in $\mathbb{R}[s]<0$, then $[2]$

$$
\begin{aligned}
& G(s) \text { is passive } \Longleftrightarrow \lambda_{\text {min }}\left[G(j \omega)+G^{*}(j \omega)\right] \geq 0 \\
& \forall \omega \in(-\infty, \infty),
\end{aligned}
$$

where $G^{*}(j \omega)$ is the conjugated transpose of $G(j \omega)$. 
Unfortunately, as it is shown in this paper, the above conjecture is wrong. Even when the open loop MIMO control system satisfies the passivity condition, this does not necessarily result in a robust control system.

In the following sections, three cases of passivation of $2 \times 2$ linear MIMO systems are presented. The results show that in spite of passivating the systems the resulting control systems are fragile, that is, nonrobust. In addition, the cause for the lack of robustness is exposed using the multivariable structure function (MSF) [5] and the well-known Singular Values Analysis (SVA). For the sake of transparency the cases are based on $2 \times 2$ linear MIMO systems. However, the results obtained from these cases apply also to the general case of $M \times M$ MIMO systems. That is, the MSF applies to the general case of $M \times M$ linear MIMO systems [6].

\section{Cases of Study}

Case 1. Consider the simple $2 \times 2$ linear time invariant MIMO system of the distillation column thoroughly analysed in [7]

$$
G(s)=\frac{\left[\begin{array}{cc}
87.8 & -86.4 \\
108.2 & -109.6
\end{array}\right]}{(75 s+1)}
$$

The eigenvalues of $\left[G(j \omega)+G^{*}(j \omega)\right]$ are shown in Figure 1.

From Figure 1 it is clear that one of the eigenvalues of $\left[G(j \omega)+G^{*}(j \omega)\right]$ is negative and therefore the MIMO system of (2) is nonpassive. However, it is possible not only to passivize but also to decouple the system with the static precompensator $K_{p}$ given by

$$
K_{p}=\left[\begin{array}{ll}
0.3994 & -0.3149 \\
0.3943 & -0.3200
\end{array}\right],
$$

resulting in the passivized system $G_{p}(s)=G(s) K_{p}$ as shown in Figure 2 by the eigenvalues of $\left[G_{p}(j \omega)+G_{p}^{*}(j \omega)\right]$ :

$$
G_{p}(s)=\frac{\left[\begin{array}{ll}
1 & 0 \\
0 & 1
\end{array}\right]}{(75 s+1)} .
$$

As mentioned above, the second step in the passive control strategy is to design a passive controller, normally a PID, based on the amended or passivized system. In this case, it is possible to design a simple decentralized PI controller $K(s)$ given by

$$
K(s)=\frac{\left[\begin{array}{cc}
(s+0.01333) & 0 \\
0 & (2 s+0.02667)
\end{array}\right]}{(0.01333 s)},
$$

resulting in the passive open loop control system $G_{o}(s)=$ $G_{p}(s) K=G(s) K_{p} K(s)$,

$$
G_{o}(s)=\frac{\left[\begin{array}{ll}
1 & 0 \\
0 & 2
\end{array}\right]}{s}
$$

with a stable closed loop matrix transfer function given by

$$
G_{\mathrm{cl}}(s)=\left[\begin{array}{cc}
\frac{1}{s+1} & 0 \\
0 & \frac{2}{s+2}
\end{array}\right] .
$$

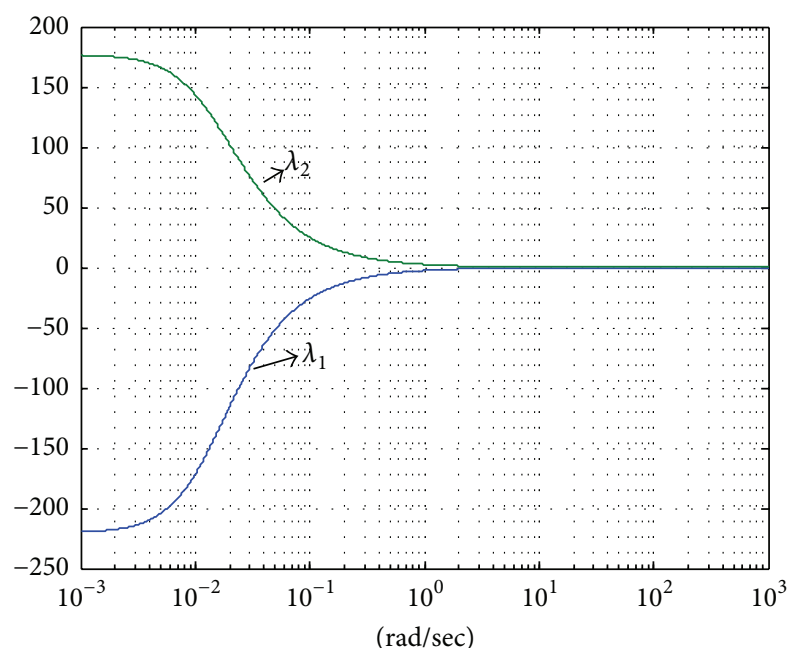

Figure 1: Eigenvalues of $\left[G(j \omega)+G^{*}(j \omega)\right]$ (Case 1).

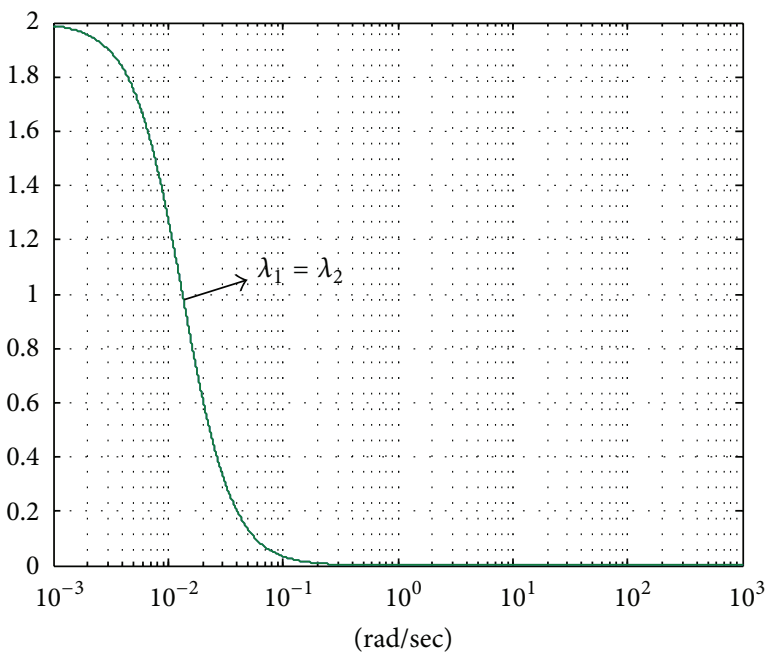

Figure 2: Eigenvalues of $\left[G_{p}(j \omega)+G_{p}^{*}(j \omega)\right]$ (Case 1).

Nonetheless, if, for instance, a small parameter perturbation or uncertainty of $+4 \%$ is introduced in the gain of the individual transfer function $g_{12}(s)$ of the system in (2) the open loop control system becomes

$$
G_{o}(s)=\frac{\left[\begin{array}{cc}
-0.3628 & 0 \\
0 & 2
\end{array}\right]}{s}
$$

which is nonpassive and closed loop unstable.

Case 2. Let the well-known quadruple tank MIMO system described by the matrix transfer function [8]

$$
\begin{aligned}
G_{2}(s) & \\
= & {\left[\begin{array}{cc}
\frac{\delta_{1} 3.533}{(63 s+1)} & \frac{\left(1-\delta_{2}\right) 3.533}{\left(2457 s^{2}+102 s+1\right)} \\
\frac{\left(1-\delta_{1}\right) 4.678}{\left(5096 s^{2}+147 s+1\right)} & \frac{\delta_{2} 4.678}{(91 s+1)}
\end{array}\right] . }
\end{aligned}
$$




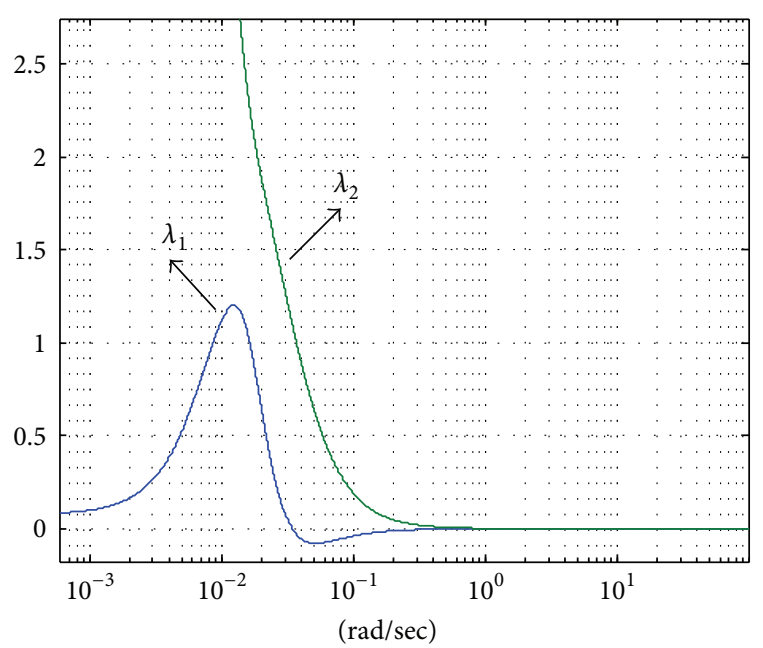

Figure 3: Eigenvalues of $\left[G_{2}(j \omega)+G_{2}^{*}(j \omega)\right]$ (Case 2).

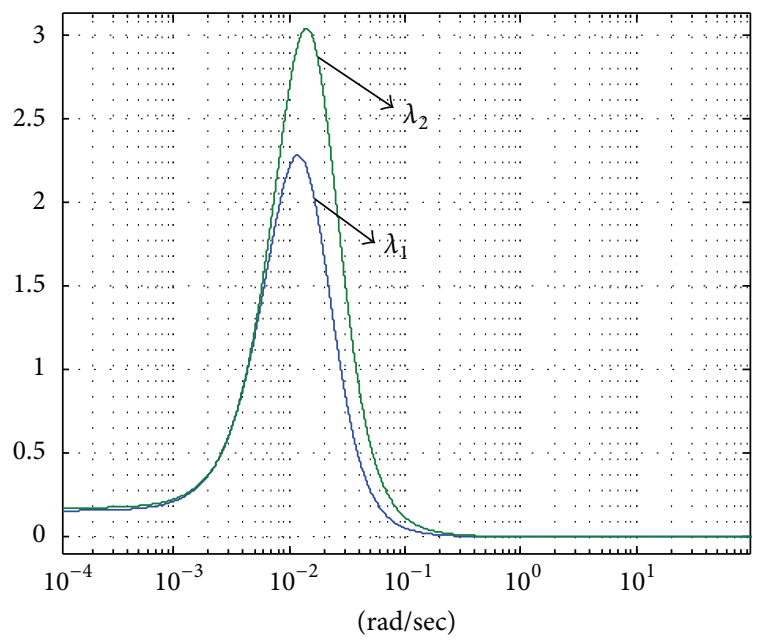

FIGURE 4: Eigenvalues of $\left[G_{p 2}(j \omega)+G_{p 2}^{*}(j \omega)\right]$ (Case 2).

If the parameters $\delta_{1}, \delta_{2} \in(0,1)$, which depend on the positions of the system valves, are set to $\delta_{1}=0.6$ and $\delta_{2}=0.41$, this yields a system for which the eigenvalues of $\left[G_{2}(j \omega)+G_{2}^{*}(j \omega)\right]$ show that the quadruple tank is nonpassive (Figure 3).

Following the strategy of Case 1 the system can be decoupled by the precompensator:

$$
\begin{aligned}
& K_{p 2}(s) \\
& =\left[\begin{array}{cc}
1 & -170.3 s-1.871 \\
\frac{-131.3 s-2.084}{5208 s^{2}+216.2 s+2.119} & 9774 s^{2}+281.9 s+1.918 \\
1
\end{array}\right.
\end{aligned}
$$

resulting in the decoupled system $G_{p 2}(s)=G_{2}(s) K_{p 2}(s)$. The eigenvalues of $\left[G_{p 2}(j \omega)+G_{p 2}^{*}(j \omega)\right]$ (Figure 4$)$ show that the precompensated system $G_{p 2}(s)$ is also passive.

Therefore, it is possible to design the decentralized PI controller $K_{2}(s)$ to the passivized system $G_{p 2}(s)$ to

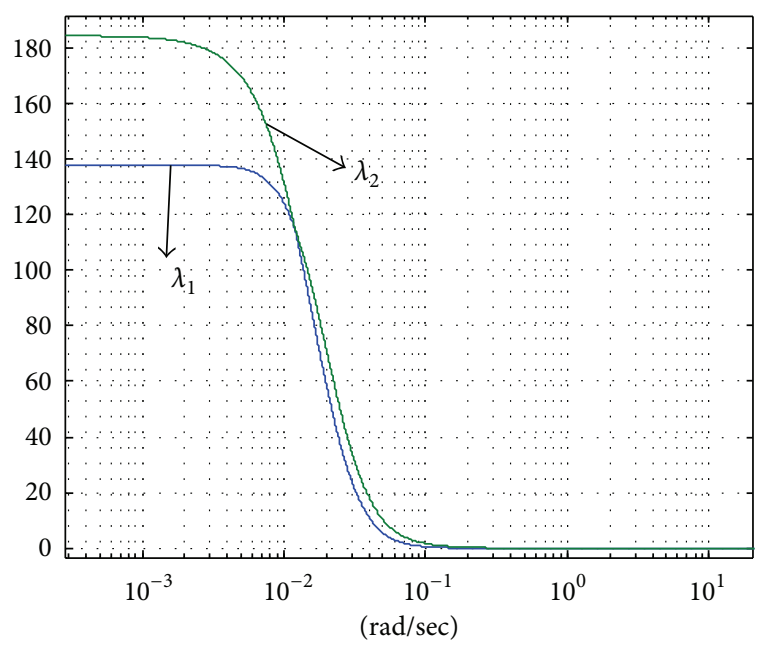

FIgURE 5: Eigenvalues of $\left[G_{o 2}(j \omega)+G_{o 2}^{*}(j \omega)\right]$ (Case 2).

obtain the open loop system $G_{o 2}(s)=G_{p 2}(s) K_{2}(s)=$ $G_{2}(s) K_{p 2}(s) K_{2}(s)$ with

$$
K_{2}(s)=\frac{\left[\begin{array}{cc}
0.35(100 s+1) & 0 \\
0 & 0.525(100 s+1)
\end{array}\right]}{s} .
$$

The eigenvalues of $\left[G_{o 2}(j \omega)+G_{o 2}^{*}(j \omega)\right]$ (Figure 5) confirm that also the open loop system $G_{o 2}(s)$ is passive. Thus, the closed loop system is stable and passive.

However, despite the passivity of the system, it can be destabilized by a small perturbation. That is, a perturbation or uncertainty of $+5 \%$ in the gain of the individual transfer function $g_{12}(s)$ of the quadruple tank destabilizes the closed loop system introducing a pair of unstable poles at $\{0.00074,0.000073\}$.

Case 3. Let $2 \times 2$ MIMO system described by the matrix transfer function $G_{3}(s)$

$$
G_{3}(s)=\frac{\left[\begin{array}{ll}
(8.6 s+8.6) & (90 s+0.9) \\
(0.05 s+1) & \left(0.1215 s^{2}+3.893 s+7.3\right)
\end{array}\right]}{\left(0.03031 s^{3}+0.5304 s^{2}+2.258 s+1\right)} .
$$

Similar to two previous cases, the eigenvalues of $\left[G_{3}(j \omega)+\right.$ $G_{3}^{*}(j \omega)$ ] (Figure 6) show that $G_{3}(s)$ is nonpassive.

Continuing with the common multivariable control strategy of decoupling via precompensation, the system $G_{3}(s)$ can be decoupled by the precompensator $K_{p 3}(s)$ given by

$$
\begin{aligned}
& K_{p 3}(s) \\
& =\left[\begin{array}{cc}
1 & \frac{(-10.47 s-0.1047)}{(s+1)} \\
\frac{(-0.4114 s-8.227)}{\left(s^{2}+32.03 s+60.06\right)} & 1
\end{array}\right] .
\end{aligned}
$$

Contrary to the two previous examples the precompensated and decoupled system $G_{p 3}(s)=G_{3}(s) K_{p 3}(s)$ remains nonpassive because the eigenvalues of $\left[G_{p 3}(j \omega)+G_{p 3}^{*}(j \omega)\right]$ are not positive at all frequencies as it is shown in Figure 7. 


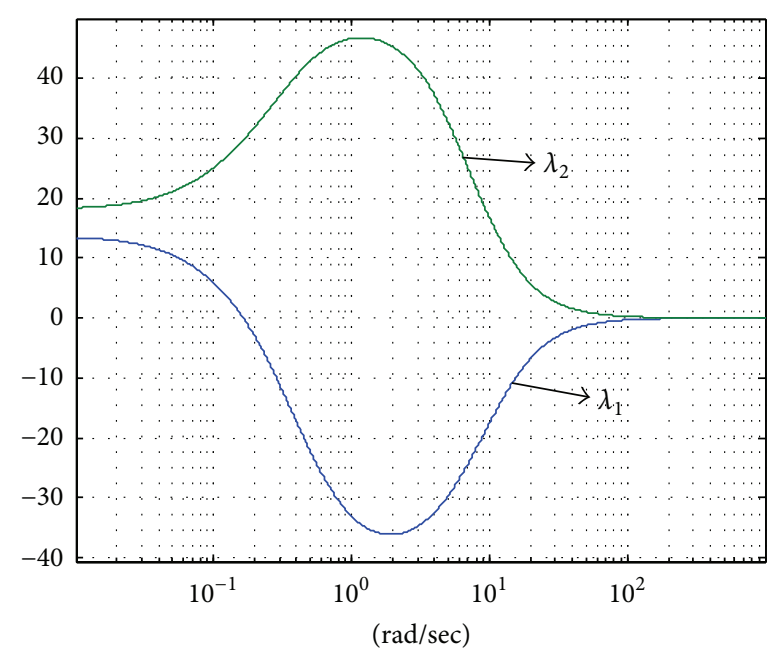

FigUre 6: Eigenvalues of $\left[G_{3}(j \omega)+G_{3}^{*}(j \omega)\right]$ (Case 3).

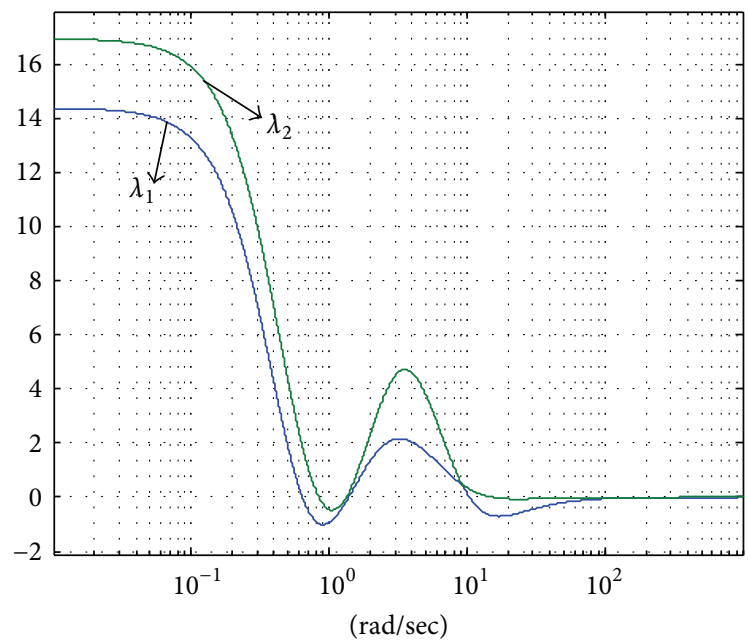

FIgUre 7: Eigenvalues of $\left[G_{p 3}(j \omega)+G_{p 3}^{*}(j \omega)\right]$ (Case 3).

Nonetheless, it is possible to both passivize and control the decoupled system $G_{3 p}(s)$ by the decentralized controller $K_{3}(s)$ comprising two PID + lead-compensator filters:

$$
K_{3}(s)=\left[\begin{array}{cc}
k_{1}(s) & 0 \\
0 & k_{2}(s)
\end{array}\right] \text {, }
$$

where

$$
\begin{aligned}
& k_{1}(s) \\
& =\frac{0.079 s^{4}+0.001461 s^{3}+0.007979 s^{2}+0.01311 s+0.00474}{s\left(s^{2}+0.1358 s+2.071\right)}, \\
& k_{2}(s)=\frac{7.9 s^{4}+0.1462 s^{3}+0.7268 s^{2}+0.8493 s+0.2607}{s\left(s^{3}+30.13 s^{2}+6.097 s+62.12\right)} .
\end{aligned}
$$

The system $G_{o 3}(s)=G_{p 3}(s) K_{3}(s)=G_{3}(s) K_{p 3}(s) K_{3}(s)$ satisfies the passivity condition as the eigenvalues of $\left[G_{o 3}(j \omega)+\right.$ $\left.G_{o 3}^{*}(j \omega)\right]$ (Figure 8) are positive at all frequencies. Consequently, the closed loop system is passive and stable.

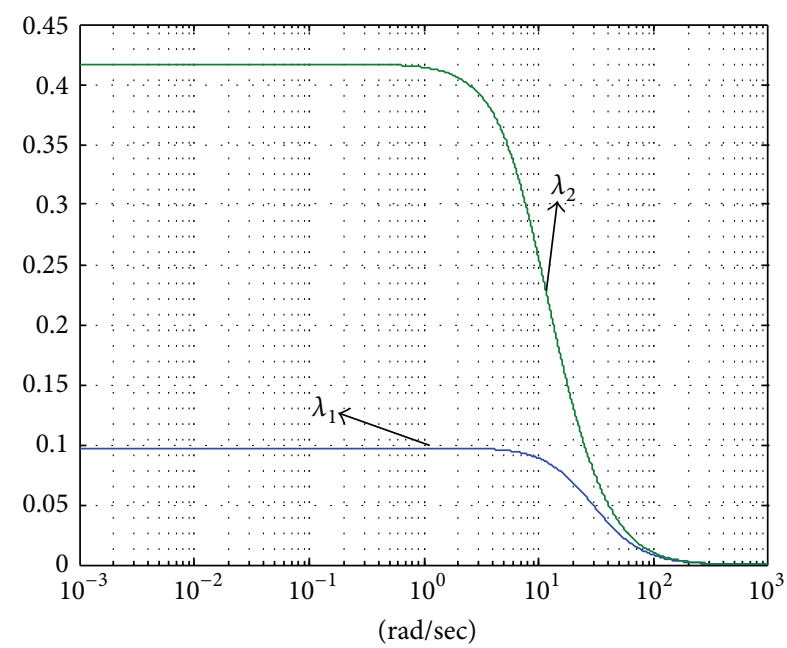

Figure 8: Eigenvalues of $\left[G_{o 3}(j \omega)+G_{o 3}^{*}(j \omega)\right]$ (Case 3).

Similar to the two preceding cases, regardless of the passivity condition of the open loop control system, its closed loop configuration can be easily destabilized, for instance, a small perturbation in the gain of the individual transfer function $(+5 \%) g_{12}(s)$ of the original system. This perturbation introduces a pair of closed loop unstable poles at $\{0.0071 \pm 1.4345 j\}$.

The cases presented prove that for multivariable control systems passivity may not necessarily imply robustness. That is, a multivariable control system may remain highly sensitive and fragile to parametric perturbations despite having been passivized.

In the next section via the multivariable structure function (MSF) this situation can be easily clarified.

\section{Robustness Analysis}

The MSF is a key element of the framework of analysis and design for linear MIMO control systems known as Individual Channel Analysis and Design (ICAD) [9-11]. ICAD allows the application of the well proved SISO classical control theory in the analysis and design of linear MIMO control systems. An explanation of ICAD is out of the scope of this paper; however, a comprehensive description and applications of ICAD can be found in [12-14].

Let $2 \times 2$ MIMO system described by the matrix transfer function

$$
G(s)=\left[\begin{array}{ll}
g_{11}(s) & g_{12}(s) \\
g_{21}(s) & g_{22}(s)
\end{array}\right] .
$$

Then, the MSF $\gamma(s)$ of $G(s)$ is defined by

$$
\gamma(s)=\frac{g_{12}(s) g_{21}(s)}{g_{11}(s) g_{22}(s)} .
$$

In [5], it was proved that $2 \times 2$ MIMO system is highly sensitive to parametric uncertainties or perturbations and nonmodeled dynamics if the MSF $\gamma(j \omega)$ is close to the point $(1,0)$ at some range of frequencies $\omega \in\left(\omega_{0}, \omega_{1}\right)$. This condition is 


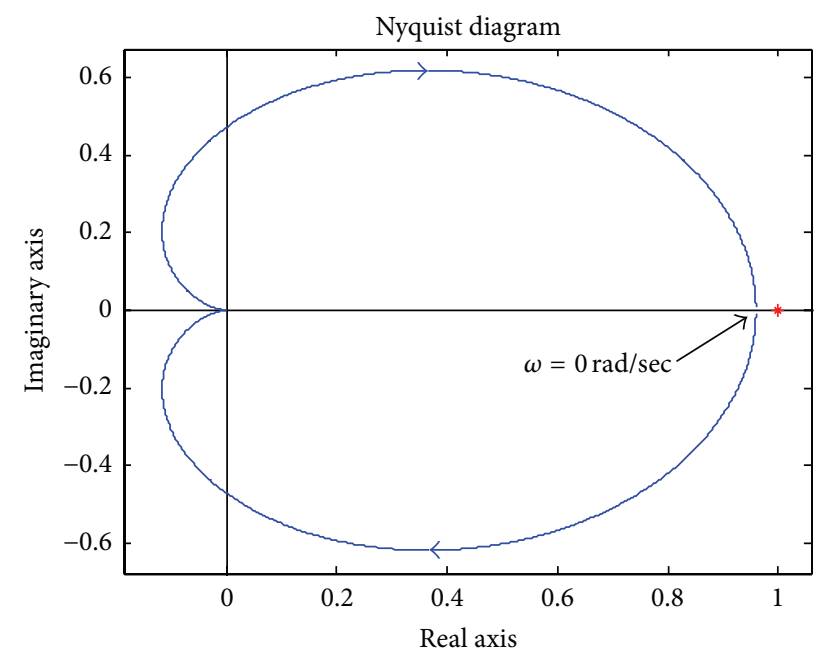

FIGURE 9: Nyquist plot of $\gamma_{2}(s)$ (Case 2).

known within ICAD as structurally nonrobust. This indicates that the number of minimum and nonminimum phase zeros can be modified by uncertainties or perturbations. Therefore, via the MSF, it is possible to determine the possibilities of a MIMO control system to satisfy the requirements of design and the conditions the controller must satisfy to comply with these requirements ensuring appropriate robustness.

Case 1 (robust analysis). The system $G(s)$ of (2) has no transmission zeros and its $\operatorname{MSF} \gamma(s)$ is given by

$$
\gamma(s)=\frac{g_{12}(s) g_{21}(s)}{g_{11}(s) g_{22}(s)}=0.9715 \text {. }
$$

Hence, the system of (2) is highly sensitive at all frequencies due to the closeness of its MSF to point $(1,0)$. This condition can likewise be explained via the condition number $\kappa(j \omega)$ :

$$
\kappa(G(j \omega))=\frac{\sigma_{\max }(G(j \omega))}{\sigma_{\min }(G(j \omega))},
$$

where $\sigma_{\max }(G(j \omega))$ and $\sigma_{\max }(G(j \omega))$ are the maximum and minimum Singular Values (SV) of $G(s)$.

The condition number $\kappa(j \omega)$ for the system of (2) is $\kappa(j \omega)=141.732$. That is, the system is ill-conditioned at all frequencies.

Case 2 (robust analysis). The system $G_{2}(s)$ of (9) is minimum phase with transmission zeros at $\{-0.0431,-0.0004$, $-0.011,-0.159\}$. On the other hand, the Nyquist plot of the MSF $\gamma_{2}(s)$ of $G_{2}(s)$ is shown in Figure 9.

From Figure 9, it is clear that $\gamma_{2}(j \omega)$ is close to the point $(1,0)$ at $\omega=0 \mathrm{rad} / \mathrm{sec}$. Hence, the system $G_{2}(s)$ is highly sensitive or ill-conditioned at $\omega=0 \mathrm{rad} / \mathrm{sec}$ as shown in Figure 10 by its condition number $\kappa_{2}(j \omega)$.

It is due to this condition that when the small perturbation of $+5 \%$ was introduced in the individual transfer function $g_{12}(s), G_{2}(s)$ became non-minimum phase with a zero at +0.000076 and consequently the closed loop control system becomes unstable.
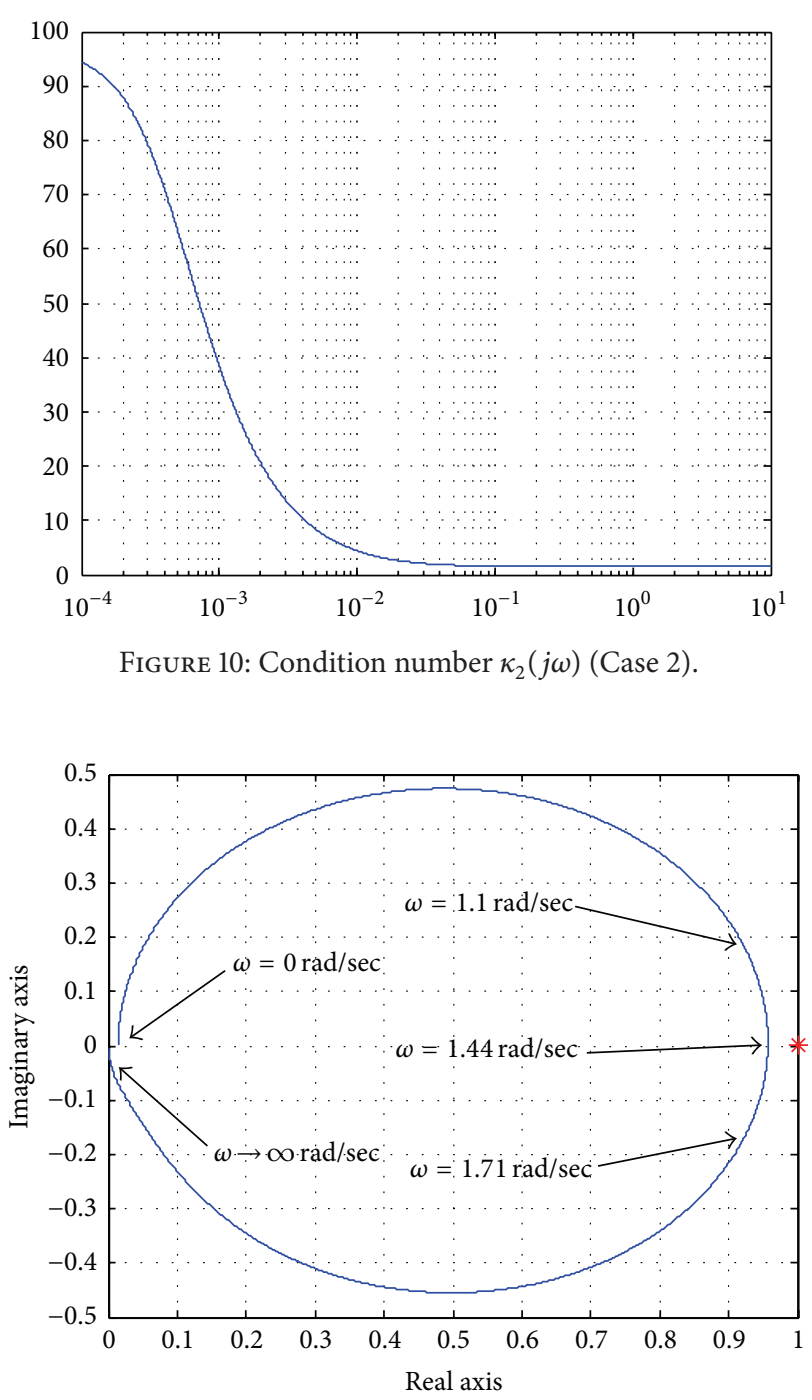

FIgURE 11: Nyquist plot of $\gamma_{3}(s)$ (Case 3).

Case 3 (robust analysis). System $G_{3}(s)$ of (12) is minimum phase with zeros at $\{-28.58,-0.0 .0677 \pm 1.437 j\}$. The Nyquist plot of the MSF $\gamma_{3}(s)$, depicted in Figure 11, indicates that $G_{3}(s)$ is highly sensitive at frequencies around $\omega=$ $1.44 \mathrm{rad} / \mathrm{sec}$.

This situation is confirmed by the plot of the condition number $\kappa_{3}(j \omega)$ (Figure 12) which clearly shows that the system is ill-conditioned in a region of frequencies around $\omega=1.44 \mathrm{rad} / \mathrm{sec}$. This explains why the closed loop system was destabilized by the small perturbation of $+5 \%$ in the gain of the individual transfer function $g_{12}(s)$. That is, due to this perturbation, the system became non-minimum phase with a pair of zeros at $\{0.0076 \pm 1.44 j\}$ and as a result, like the previous case, closed loop unstable.

\section{Conclusions}

In this paper, it is shown that in the case of linear MIMO control systems passivity does not necessarily imply good robustness properties. This was exemplified by means of 


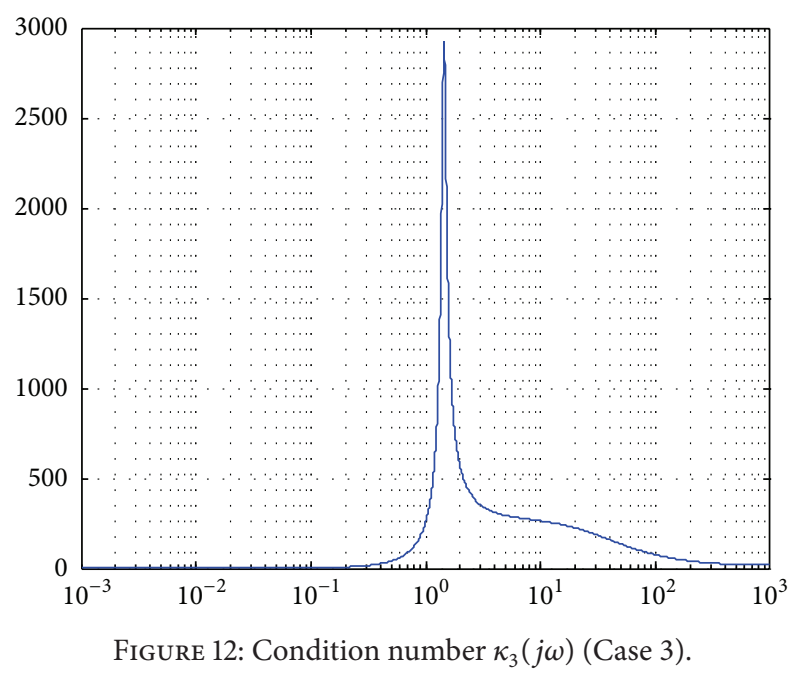

3 cases where despite the passivation of the open loop control systems it was possible to destabilize their closed loops configurations by small parametric perturbations. That is, the control systems are highly sensitive regardless of its passive condition. This situation was exposed via the multivariable structure function and the condition number. In particular, it was shown that the lack of structural robustness, illconditioning, cannot be amended via passivation. The three cases of study represent the three possible conditions in which a system is not structurally robust or ill-posed: at all frequencies, at one particular frequency, and along a region of frequencies.

It can be concluded that in order to exploit all the benefits of passivity in MIMO control systems it is necessary to consider the lack of structural robustness or ill-conditioning of the process before attempting any passivation strategy.

Finally, it is possible to extend the application of the multivariable structure function to the passive analysis of the general case of $M \times M$ MIMO systems.

\section{Conflict of Interests}

The authors declare that there is no conflict of interests related to this paper.

\section{References}

[1] E. Licéaga-Castro, J. Licéaga-Castro, C. E. Ugalde-Loo, and E. M. Navarro-López, "Efficient multivariable submarine depthcontrol system design," Ocean Engineering, vol. 35, no. 17-18, pp. 1747-1758, 2008.

[2] B. Brogliato, R. Lozano, B. Maschke, and O. Egeland, Dissipative Systems Analysis and Control. Theory and Applications, Communications and Control Engineering Series, Springer, London, UK, 2nd edition, 2007.

[3] A. G. Kelkar and S. M. Joshi, "Robust control of non-passive systems via passification," in Proceedings of the American Control Conference, vol. 5, pp. 2657-2661, IEEE, Albuquerque, NM, USA, June 1997.
[4] A. G. Kelkar and S. M. Joshi, "Robust passification and control of non-passive systems," in Proceedings of the American Control Conference, pp. 3133-3137, Philadelphia, Pa, USA, June 1998.

[5] J. O’Reilly and W. E. Leithead, "Multivariable control by 'individual channel design,' International Journal of Control, vol. 54, no. 1, pp. 1-46, 1991.

[6] W. E. Leithead and J. O’Reilly, " $m$-input $m$-output feedback control by individual channel design part 1 . Structural issues," International Journal of Control, vol. 56, no. 6, pp. 1347-1397, 1992.

[7] S. Skogestad and I. Postlethwaite, Multivariable Feedback Control: Analysis and Design, Wiley, 2007.

[8] K. H. Johansson, "The quadruple-tank process: a multivariable laboratory process with an adjustable zero," IEEE Transactions on Control Systems Technology, vol. 8, no. 3, pp. 456-465, 2000.

[9] W. E. Leithead and J. O'Reilly, "Performance issues in the individual channel design of 2-input 2-output systems. Part 1. Structural issues," International Journal of Control, vol. 54, no. 1 , pp. 47-82, 1991.

[10] W. E. Leithead and J. O'Reilly, "Performance issues in the individual channel design of 2-input 2-output systems. Part 2. Robustness issues," International Journal of Control, vol. 55, no. 1, pp. 3-47, 1992.

[11] W. E. Leithead and J. O'Reilly, "Performance issues in the individual channel design of 2-input 2-output systems, Part 3. Non-diagonal control and related issues," International Journal of Control, vol. 55, no. 1, pp. 265-312, 1992.

[12] E. Licéaga-Castro, J. Licéaga-Castro, and C. E. Ugalde-Loo, "Beyond the existence of diagonal controllers: from the relative gain array to the multivariable structure function," in Proceedings of the 44th IEEE Conference on Decision and Control, and the European Control Conference (CDC-ECC '05), pp. 71507156, Sevilla, Spain, December 2005.

[13] E. Licéaga-Castro, C. E. Ugalde-Loo, J. Licéaga-Castro, and P. Ponce, "An efficient controller for SV-PWM VSI based on the multivariable structure function," in Proceedings of the 44th IEEE Conference on Decision and Control, and the European Control Conference (CDC-ECC '05), pp. 4754-4759, IEEE, Sevilla, Spain, December 2005.

[14] L. Amezquita-Brooks, J. Liceaga-Castro, and E. Liceaga-Castro, "Speed and position controllers using indirect field-oriented control: a classical control approach," IEEE Transactions on Industrial Electronics, vol. 61, no. 4, pp. 1928-1943, 2014. 

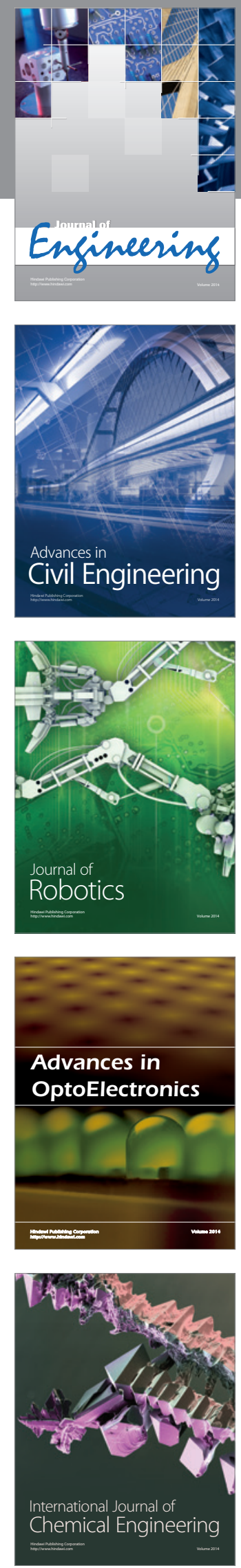

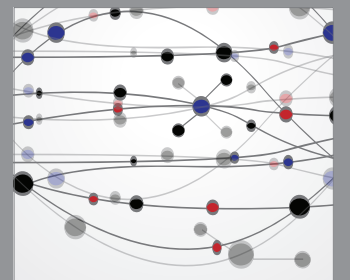

The Scientific World Journal
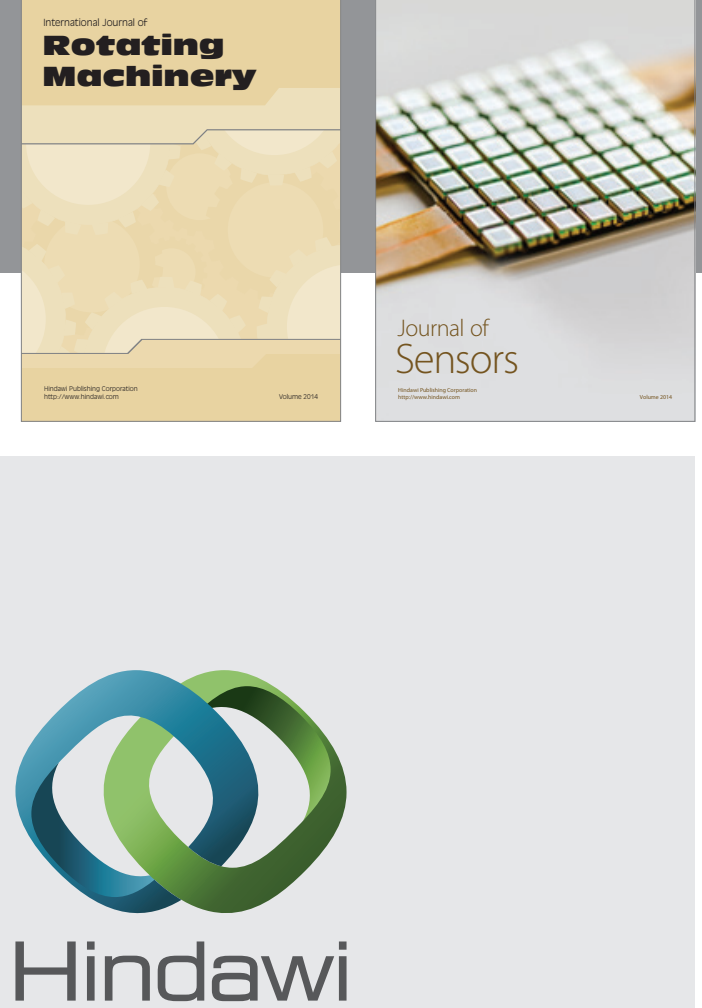

Submit your manuscripts at http://www.hindawi.com
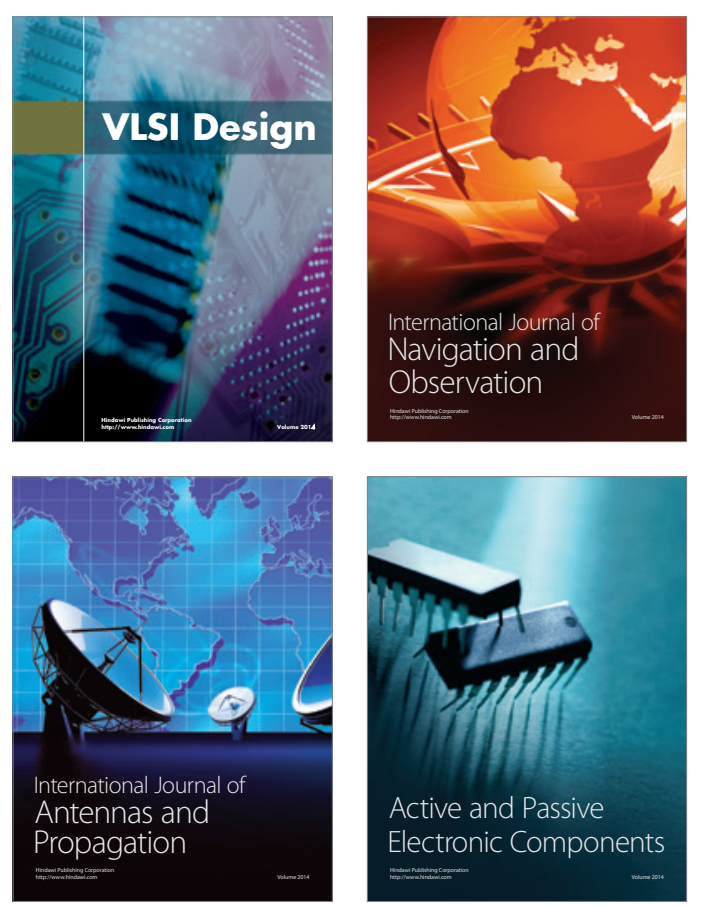
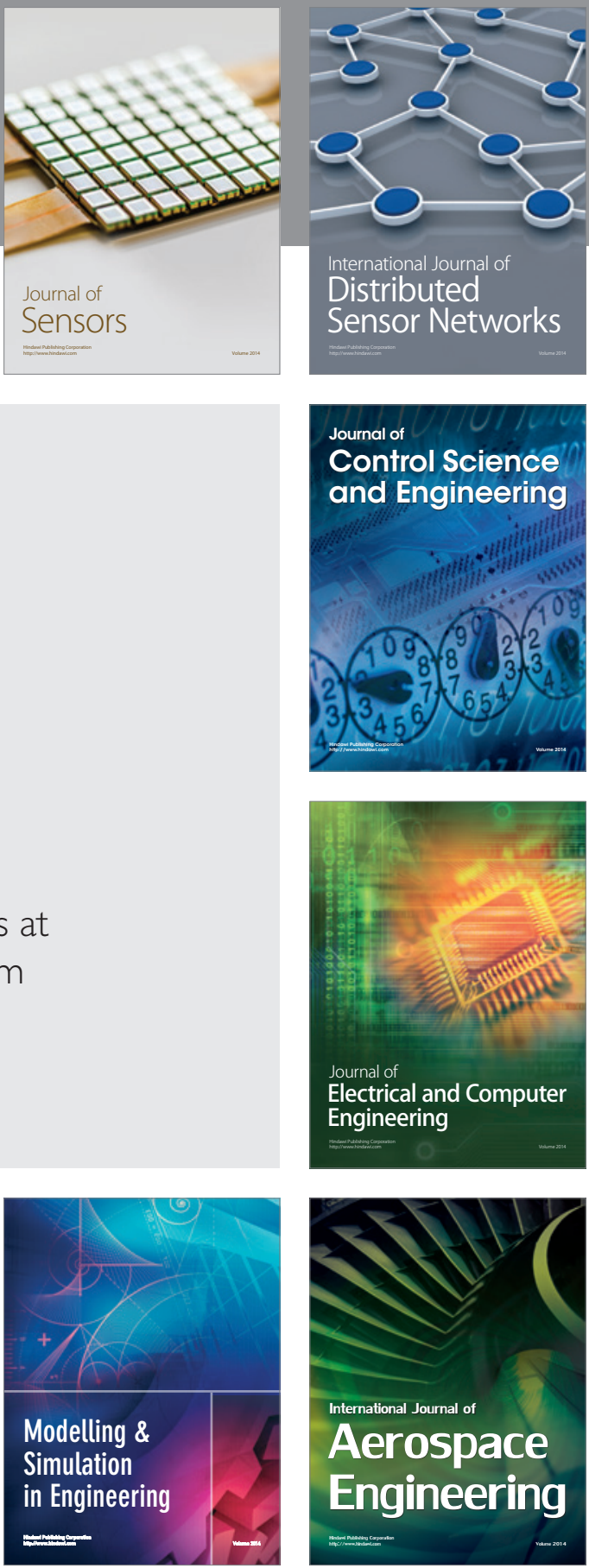

Journal of

Control Science

and Engineering
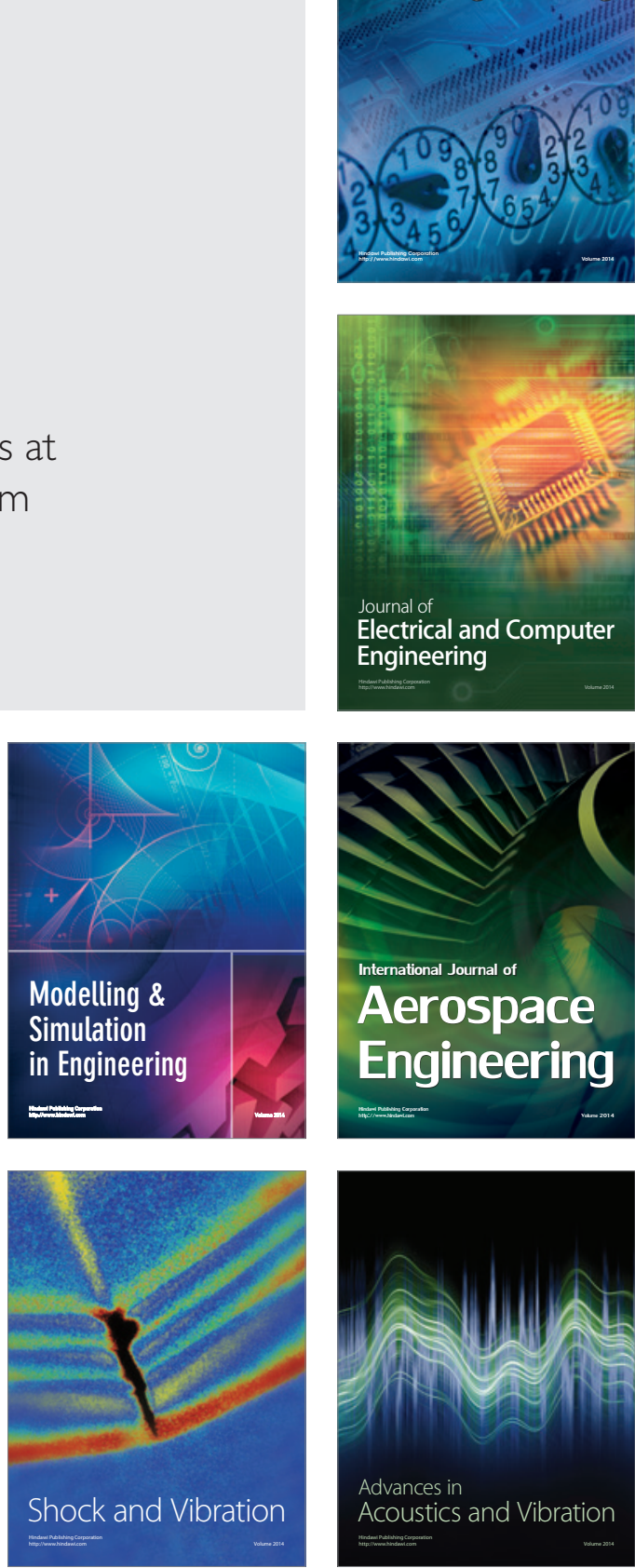